\title{
Unplanned 30-Day Hospital Readmissions of Symptomatic Carotid and Vertebral Artery Dissection
}

\author{
Tapan Mehta, ${ }^{\text {a }}$ Smit Patel, ${ }^{\mathrm{b}}$ Shailesh Male, ${ }^{\mathrm{a}}$ Romil Parikh, ${ }^{\mathrm{c}}$ Kathan Mehta, ${ }^{\mathrm{d}}$ Kamakshi Lakshminarayan, ${ }^{\mathrm{a}, \mathrm{c}}$ \\ Ramachandra Tummala, ${ }^{\mathrm{e}}$ Mustapha Ezzeddine ${ }^{\mathrm{a}}$ \\ aDepartment of Neurology, University of Minnesota, Minneapolis, MN, USA \\ ${ }^{b}$ Department of Neurology, University of Connecticut, Farmington, CT, USA \\ 'Division of Epidemiology and Community Health, University of Minnesota, Minneapolis, MN, USA \\ ${ }^{d}$ Division of Hematology-Oncology, University of Pittsburgh School of Medicine, Pittsburgh, PA, USA \\ 'Department of Neurosurgery, University of Minnesota, Minneapolis, MN, USA
}

\section{Dear Sir:}

Cervical artery dissection in young adults accounts for $10 \%$ to $25 \%$ of all ischemic strokes. Sample size is one of the foremost limitations delineated in a majority of the studies evaluating recurrent risk of stroke due to cervical artery dissection. ${ }^{1}$

We used the 2014 Nationwide Readmission Database to analyze 30-day unplanned readmissions (30RR) for symptomatic carotid artery dissection (SCAD) (weighted $n=2,686$ ) and symptomatic vertebral artery dissection (SVAD) (weighted $n=2,837$ ). ${ }^{2}$ Differences in demographic characteristics of both the groups based on the relevant available variables in the database and International Classification of Diseases, Ninth Revision (ICD-9) diagnosis codes are described in Table 1. Readmission trends are depicted in Figure 1. Top causes of unplanned readmissions are described in Table 2.

Overall 30RR for SCAD was not significantly higher than SVAD ( $9.08 \%$ vs. $8.43 \%, P=0.412)$. Overall unplanned readmissions due to ischemic strokes in our study are similar to the findings of the CADISS trial1; however, 30RR due to ischemic stroke were higher in SVAD compared to SCAD (4.14\% vs. $1.60 \%, P<0.001)$. Approximately $50 \%$ of total readmissions for first 30 days were admitted by day 7 in both SCAD and SVAD groups. ${ }^{3.4}$

Higher incidence of intracranial dissection with SVAD compared to SCAD potentially explains higher intracranial hemorrhage with SVAD (intracerebral hemorrhage: $11.62 \%$ vs. $5.41 \%$, $P<0.001$; subarachnoid hemorrhage: $3.21 \%$ vs. $0.78 \%, P<0.001) .{ }^{5}$ The incidence of intracerebral hemorrhage amongst readmitted patients was also significantly higher with SVAD $(7.9943 \%$ vs. $3.6257 \%, P=0.016$ ). These findings are less likely to be related to reperfusion therapies, as anterior circulation infarcts tend to have higher hemorrhagic transformation rates. ${ }^{6}$ We also noted a higher incidence of reperfusion therapies with carotid dissections.

Peripheral, visceral and aortic artery aneurysms were present in $11.55 \%$ patients with SCAD (related 30RR 1.16\%, third leading cause) and $4.73 \%$ patients with SVAD patients (related 30RR $0.88 \%$, second leading cause). These findings suggest that there might be some value of having a low threshold to screen these patients for additional vascular abnormalities, especially those with known connective tissue disorders.

Tertiary care centers are reported to have poorer outcomes for cervical artery dissections, as complicated cases are usually referred to such institutions. ${ }^{7}$ Our study too showed a higher readmission trend in larger size hospitals. Medicare and Medicaid as primary payer had higher rates of readmission compared to private insurance along with length of hospital stay as described in Table 1. It is important to consider that quality of access to care after discharge from hospital could be a contributing factor leading to higher readmissions in such population.

The study findings need to be taken into consideration in light of shortcomings intrinsic to secondary analysis of a large administrative database. We were not able to identify the approach for medical management during index hospitalization (i.e., anticoagulant vs. antiplatelet therapy), imaging information, location of dissection (intra vs. extracranial), and degree 
Table 1. Baseline characteristics of study population

\begin{tabular}{|c|c|c|c|c|c|c|}
\hline \multirow{2}{*}{ Variable } & \multicolumn{3}{|c|}{ Carotid dissection } & \multicolumn{3}{|c|}{ Vertebral dissection } \\
\hline & Readmit & Non-readmit & $P$ & Readmit & Non-readmit & $P$ \\
\hline Number & 244 & 2,442 & & 239 & 2,598 & \\
\hline Age & $55.6 \pm 2.0$ & $53.2 \pm 0.6$ & 0.24 & $53.1 \pm 1.8$ & $48.1 \pm 0.5$ & $<0.01$ \\
\hline Charlson's Index & $2.4 \pm 0.2$ & $2.0 \pm 0.1$ & 0.06 & $1.8 \pm 0.2$ & $1.5 \pm 0.0$ & 0.05 \\
\hline $\begin{array}{l}\text { Median household income category for patient's zip code } \\
\text { (percentile) (\%) }\end{array}$ & & & 0.41 & & & 0.95 \\
\hline $0-25$ th & 24.3 & 18.8 & & 15.0 & 16.7 & \\
\hline 26-50th & 20.1 & 24.2 & & 20.6 & 22.0 & \\
\hline 51-75th & 23.1 & 27.5 & & 31.0 & 30.6 & \\
\hline 76-100th & 32.5 & 29.4 & & 33.4 & 30.7 & \\
\hline Primary payer (\%) & & & 0.03 & & & $<0.01$ \\
\hline Medicare & 29.6 & 24.8 & & 36.7 & 18.6 & \\
\hline Medicaid & 21.6 & 12.1 & & 17.1 & 10.8 & \\
\hline Private insurance & 41.1 & 53.1 & & 39.7 & 61.8 & \\
\hline Self-pay/no charge/other & 7.7 & 9.2 & & 6.6 & 8.7 & \\
\hline Length of hospital stay in days (LOS) & & & $<0.01$ & & & $<0.01$ \\
\hline Medicare & $8.8 \pm 1.7$ & $7.3 \pm 0.5$ & & $7.9 \pm 1.1$ & $7.7 \pm 0.6$ & \\
\hline Medicaid & $14.4 \pm 4.6$ & $12.7 \pm 1.4$ & & $10.1 \pm 2.9$ & $7.5 \pm 0.7$ & \\
\hline Private insurance & $8.8 \pm 1.7$ & $7.2 \pm 0.4$ & & $4.3 \pm 0.6$ & $6.1 \pm 0.4$ & \\
\hline Self-pay/no charge/other & $3.2 \pm 0.7$ & $9.7 \pm 1.8$ & & $9.7 \pm 3.5$ & $7.7 \pm 1.8$ & \\
\hline Hospital bed size & & & 0.01 & & & 0.21 \\
\hline Small & 4.5 & 7.7 & & 3.7 & 8.1 & \\
\hline Medium & 30.4 & 17.4 & & 20.9 & 20.9 & \\
\hline Large & 65.1 & 74.9 & & 75.4 & 71.0 & \\
\hline Admission type (\%) & & & 0.46 & & & 0.92 \\
\hline Non-elective & 92.8 & 90.2 & & 95.5 & 95.2 & \\
\hline Elective & 7.2 & 9.8 & & 4.5 & 4.8 & \\
\hline Admission day (\%) & & & 0.89 & & & 0.88 \\
\hline Weekdays & 74.8 & 75.6 & & 76.0 & 76.6 & \\
\hline Weekend & 25.3 & 24.4 & & 24.1 & 23.4 & \\
\hline Disposition (\%) & & & 0.01 & & & $<0.01$ \\
\hline Home & 55.1 & 71.2 & & 56.7 & 74.2 & \\
\hline Home health care & 11.9 & 8.3 & & 9.9 & 10.5 & \\
\hline Facility & 31.4 & 19.9 & & 32.5 & 15.0 & \\
\hline Against medical advice & 1.6 & 0.6 & & 0.9 & 0.3 & \\
\hline \multicolumn{7}{|l|}{ Comorbidities (\%) } \\
\hline Hypertension & 60.5 & 51.0 & 0.07 & 61.6 & 59.1 & 0.65 \\
\hline Hyperlipidemia & 33.2 & 42.0 & 0.16 & 36.5 & 38.4 & 0.68 \\
\hline Atrial fibrillation & 13.8 & 4.1 & 0.01 & 10.5 & 8.5 & 0.51 \\
\hline Smoking & 17.5 & 17.8 & 0.93 & 23.7 & 21.0 & 0.62 \\
\hline Heart failure & 4.8 & 2.7 & 0.22 & 3.8 & 4.1 & 0.89 \\
\hline Ischemic heart disease & 13.4 & 8.5 & 0.13 & 16.5 & 10.9 & 0.08 \\
\hline Atherosclerosis & 3.5 & 1.0 & 0.06 & 2.3 & 1.4 & 0.42 \\
\hline Diseases of endocardium & 3.5 & 2.6 & 0.63 & 4.4 & 3.3 & 0.63 \\
\hline Diabetes & 14.3 & 14.3 & 0.99 & 20.8 & 14.1 & 0.1 \\
\hline Intracerebral hemorrhage & 3.4 & 2.0 & 0.32 & 8.0 & 3.6 & 0.02 \\
\hline Hypercoagulable state & 0.8 & 1.0 & 0.8 & 1.6 & 1.5 & 0.96 \\
\hline
\end{tabular}


Table 1. Continued

\begin{tabular}{|c|c|c|c|c|c|c|}
\hline \multirow{2}{*}{ Variable } & \multicolumn{3}{|c|}{ Carotid dissection } & \multicolumn{3}{|c|}{ Vertebral dissection } \\
\hline & Readmit & Non-readmit & $P$ & Readmit & Non-readmit & $P$ \\
\hline Subarachnoid hemorrhage & 0.0 & 0.8 & & 2.2 & 1.0 & 0.47 \\
\hline Trauma related injury to blood vessel of head and neck & 0.7 & 0.5 & 0.8 & 0.8 & 0.4 & 0.55 \\
\hline Unruptured aneurysm (aortic, peripheral) & 7.7 & 3.9 & 0.23 & 2.0 & 2.8 & 0.55 \\
\hline Intravenous thrombolytic use & 15.5 & 12.1 & 0.34 & 6.0 & 4.9 & 0.71 \\
\hline Mechanical thrombectomy & 8.3 & 5.2 & 0.3 & 3.7 & 1.1 & 0.06 \\
\hline $\begin{array}{l}\text { Endovascular procedure other than mechanical thrombec- } \\
\text { tomy }\end{array}$ & 5.1 & 2.6 & 0.08 & 3.2 & 2.4 & 0.41 \\
\hline
\end{tabular}

Values are presented as mean \pm standard deviation or number (\%). Unpaired t-test was used for continuous variables, and chi-square test or Fisher's exact test was used for categorical variables.

Table 2. Top causes of 30RR in carotid and vertebral artery dissection

\begin{tabular}{|c|c|c|c|c|c|c|}
\hline \multirow[b]{2}{*}{ No. } & \multicolumn{3}{|c|}{ Carotid dissection } & \multicolumn{3}{|l|}{ Vertebral dissection } \\
\hline & Causes & $\begin{array}{c}\text { Cause specific } \\
\text { 30RR }\end{array}$ & $\begin{array}{c}\text { Total } \\
\text { readmissions } \\
(\%)\end{array}$ & Causes & $\begin{array}{l}\text { Cause specific } \\
\text { 30RR }\end{array}$ & $\begin{array}{c}\text { Total } \\
\text { readmissions } \\
(\%)\end{array}$ \\
\hline 1 & Ischemic stroke & 1.6 & 10.4 & Ischemic stroke & 4.1 & 30.8 \\
\hline 2 & Intracranial hemorrhage & 1.3 & 8.2 & Aortic; peripheral; and visceral artery aneurysms & 0.9 & 6.6 \\
\hline 3 & $\begin{array}{l}\text { Aortic; peripheral; and visceral artery } \\
\text { aneurysms }\end{array}$ & 1.2 & 7.5 & Late effects of cerebrovascular disease & 0.8 & 5.9 \\
\hline 4 & Retinal disorders & 0.9 & 5.5 & Connective tissue disease & 0.4 & 3.2 \\
\hline 5 & Transient cerebral ischemia & 0.7 & 4.6 & Occlusion or stenosis of precerebral arteries & 0.4 & 2.8 \\
\hline 6 & Intestinal infection & 0.6 & 4.0 & Headache & 0.4 & 2.8 \\
\hline 7 & Venous embolism and thrombosis & 0.6 & 4.0 & Gram negative septicemia & 0.4 & 2.8 \\
\hline 8 & Nonspecific chest pain & 0.6 & 3.6 & Unspecified septicemia & 0.4 & 2.8 \\
\hline 9 & Unspecified septicemia & 0.5 & 3.2 & $\begin{array}{l}\text { Nervous system symptoms and disorders other } \\
\text { than stroke }\end{array}$ & 0.3 & 2.4 \\
\hline 10 & $\begin{array}{l}\text { Nervous system symptoms and disor- } \\
\text { ders other than stroke }\end{array}$ & 0.4 & 2.4 & Nonmalignant breast conditions & 0.3 & 2.2 \\
\hline
\end{tabular}

30RR, 30-day unplanned readmission.

- Carotid - Vertebral

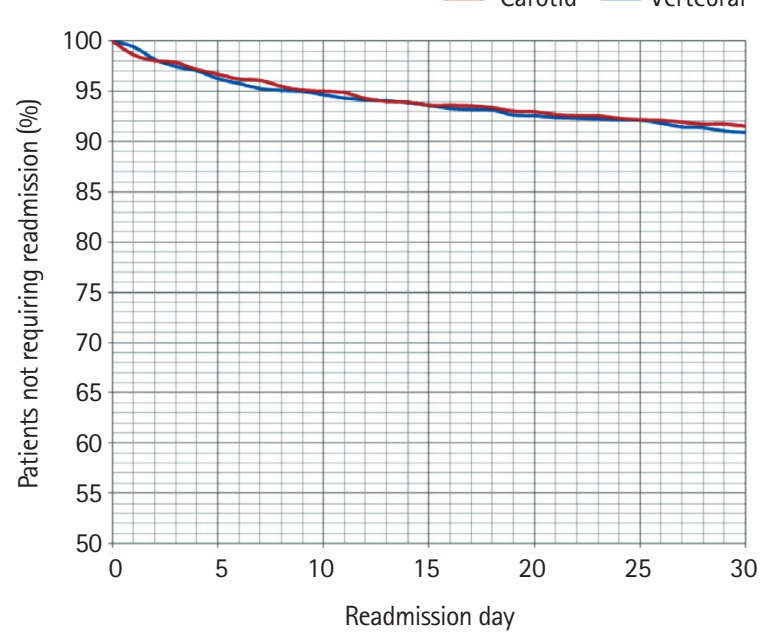

Figure 1. Readmission trends for symptomatic carotid and vertebral artery dissection. of vessel stenosis. Knowledge of these point of care clinical information would certainly further help identify the root causes of differences in readmission risk.

Despite these limitations, our study represents one of the largest cohort of symptomatic cervical artery dissection cases and contributes to current understanding of primary etiologies and demographic differences of 30 days readmissions. It shows that overall readmission rates are not significantly different for SCAD and SVAD. However, patients with vertebral artery dissections have higher rates of readmission with ischemic stroke and also are more likely to have intracranial hemorrhage.

\section{References}

1. CADISS trial investigators, Markus HS, Hayter E, Levi C, Feldman $A$, Venables $G$, et al. Antiplatelet treatment compared 
with anticoagulation treatment for cervical artery dissection (CADISS): a randomised trial. Lancet Neurol 2015;14:361-367.

2. Healthcare Cost and Utilization Project (HCUP): Nationwide Readmissions Database. Agency for Healthcare Research and Quality. https://www.ahrq.gov/data/hcup/index.html. 2013. Accessed September 14, 2018.

3. Arauz A, Ruiz A, Pacheco G, Rojas P, Rodríguez-Armida M, Cantú

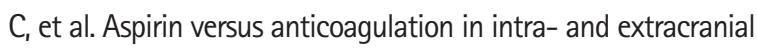
vertebral artery dissection. Eur J Neurol 2013;20:167-172.

4. Kennedy F, Lanfranconi S, Hicks C, Reid J, Gompertz P, Price $C_{\text {, et }}$ al. Antiplatelets vs anticoagulation for dissection: CADISS nonrandomized arm and meta-analysis. Neurology 2012;79:686689.

5. Debette $\mathrm{S}$, Compter $\mathrm{A}$, Labeyrie MA, Uyttenboogaart M, Metso TM, Majersik JJ, et al. Epidemiology, pathophysiology, diagnosis, and management of intracranial artery dissection. Lancet Neurol 2015;14:640-654.

6. Terruso V, D'Amelio M, Di Benedetto N, Lupo I, Saia V, Famoso
$\mathrm{G}$, et al. Frequency and determinants for hemorrhagic transformation of cerebral infarction. Neuroepidemiology 2009;33: 261-265.

7. Yaghi S, Maalouf N, Keyrouz SG. Cervical artery dissection: risk factors, treatment, and outcome: a 5-year experience from a tertiary care center. Int J Neurosci 2012;122:40-44.

Correspondence: Tapan Mehta

Department of Neurology, University of Minnesota, Minneapolis, MN 55455, USA

Tel: +1-267-237-0972

Fax: +1-612-624-0687

E-mail:mehta230@umn.edu

Received: August 9, 2018

Revised: September 13, 2018

Accepted: September 14, 2018

The authors have no financial conflicts of interest. 